\title{
Information Technology for the Morphological Analysis of the Lymphoid Cell Nuclei ${ }^{1}$
}

\author{
Igor Gurevich ${ }^{1}$, Dmitry Harazishvilii ${ }^{2}$, Irina Jernova ${ }^{1}$, Andrei Khilkov ${ }^{1}$, \\ Alexey Nefyodov ${ }^{1}$, and Ivan Vorobjev ${ }^{2}$ \\ ${ }^{1}$ Scientific Council "Cybernetics" of the Russian Academy of Sciences \\ 40, Vavilov str., 119991 Moscow, GSP-1, Russian Federation \\ igourevi@ccas.ru \\ ${ }^{2}$ Hematological Scientific Center of the Russian Academy of Medical Sciences \\ 4a, Novyi Zykovskii pr., 125167 Moscow, Russian Federation \\ ivorobjev@mail.ru
}

\begin{abstract}
The new results of the research in the field of automation of hematopoietic tumor diagnostics by analysis of the images of cytological specimens are presented. The main result is a new information technology for the morphological analysis of the lymphoid cell nuclei of patients with hematopoietic tumors based on the combined use of pattern recognition and image analysis techniques. The principal characteristic of the proposed technology is that the features used for description of lymphocyte nuclei are chosen and calculated from the images of specimens by image processing and analysis methods, and also by methods of mathematical morphology and Fourier analysis. The proposed technology provides transition from the diagnostic analysis of lymphocyte nuclei to diagnosing the patients with hematopoietic tumors by means of pattern recognition techniques. Experimental check of the technology shows that it can be successfully used in program system for automated diagnostics of the hematopoietic tumors.
\end{abstract}

\section{Introduction}

In this paper, we describe new results of the research into automation of hematopoietic tumors diagnostics on the base of analysis of the images of cytological specimens. This work has been conducted since 2000 by the researchers of the Scientific Council "Cybernetics" of the Russian Academy of Sciences together with the researchers of the Hematological Scientific Center of the Russian Academy of Medical Sciences [1, 4]. The necessary condition of such automation is the development of information technology for morphological analysis of the lymphoid cell nuclei of patients with hematopoietic tumors, which could be implemented in corresponding software system

\footnotetext{
${ }^{1}$ This work was partly supported by the Russian Foundation for Basic Research (grant No. 00-0790004, 01-07-90016, and 03-07-90406) and by Federal Target-Oriented Program "Research and Development in the Priority Directions of Science and Technology" in 2002-2006 (project No. 37.0011.11.0016).
} 
for automated diagnostics. The main result of the work is an information technology for the morphological analysis of the lymphoid cell nuclei of patients with hematopoietic tumors based on the combined use of pattern recognition and image analysis techniques.

In hematooncology, the morphological analysis is a crucial element of diagnostic process and plays an important role in evaluation of its progression. The hematopoietic tumors are diagnosed according to informal and unquantifiable details of tumor cell morphology (mainly nucleal). Such kind of analysis depends mostly on hematologist's qualification and experience.

The need for increased efficiency and accuracy of the hematopoietic tumors diagnostics based on morphological analysis of the blood cells makes the development of automated diagnostic system very urgent.

The developed information technology for the morphological analysis of the cell specimens includes the following stages of data preparation and analysis:

1. Creation of the image database preparations of lymphatic tissues with isolated lymphocyte nuclei for patients with different lymphoid tumors.

2. Normalization of the images in order to compensate for different illumination conditions and different colors of stain used for the specimens.

3. Choice of features which capture morphological characteristics of lymphocytes nuclei useful for lymphoma diagnosis.

4. Extraction of diagnostically important objects and features from the images of specimens by image processing techniques.

5. Calculation of values, statistical and qualitative analysis of the chosen features for the set of available nuclei.

6. Selection of features for generating feature descriptions of lymphoma cell nuclei.

7. Cluster analysis of the nuclei by the use of the generated feature descriptions.

8. Analysis of the obtained clusters by using the criterion of entering into these clusters the cells of two groups of patients with different diagnoses.

9. Formation of a new feature space for description of the patients:

- the 'large' clusters of the cell nuclei are selected;

- the new features are relational numbers of patient's nuclei that belong to the selected clusters.

10. Diagnosing the patients by the use of efficient recognition algorithms (for example, recognition algorithms based on estimate calculation [6]) applied to the feature descriptions developed in p.9.

Sections 2-4 contain methods and means used for implementing the above stages.

\section{General Characteristic of the Source Data}

A base of photomicrographic images of lymphatic tissue imprints was created to select and describe diagnostically important features of lymphocyte nuclei images. The base contains 1585 photos of specimens of 36 patients. We choose 25 cases of aggressive lymphoid tumors (de novo large and mixed cell lymphomas and transformed chronic 
lymphocytic leukemia) and 10 cases of indolent chronic lymphocytic leukemia. In one case the reactive lymphoid hyperplasia was diagnosed.

Footprints of lymphoid organs were obtained within 20-30 min after biopsy fixed in methanol and stained by Giemsa stain. Digital images were obtained using custom made videomicroscopic system based on Leica DMRB microscope equipped with Kodak DC-290 camera and Planapo oil immersion objectives x63 or x100. The equivalent area of one pixel was 0,0036 or $0,0064 \mu^{2}$. The photos of specimens were collected and stored as the RGB-images in 24-bit TIFF format. Fig. 1 shows the monochrome picture of the slide of lymphatic gland. The dark rounded regions against the light background including the light streaks are the nuclei of lymphocytes.

The preprocessing of images compensates for different illumination conditions and different colors of stain used for the preparations. It was accomplished by obtaining the monochrome images with 256 gray levels by the rule:

$$
\operatorname{gray}(x, y)=\operatorname{green}(x, y) \frac{R}{128},
$$

where $\operatorname{gray}(x, y)$ is a gray level at the point $(x, y)$ of the monochrome image, $\operatorname{green}(x$, $y$ ) is a green value at the point $(x, y)$ of the original RGB-image, $R$ is an average red value at the points of the original RGB-image. This rule was selected experimentally, it gives us an image with good contrast and clear nuclei details.

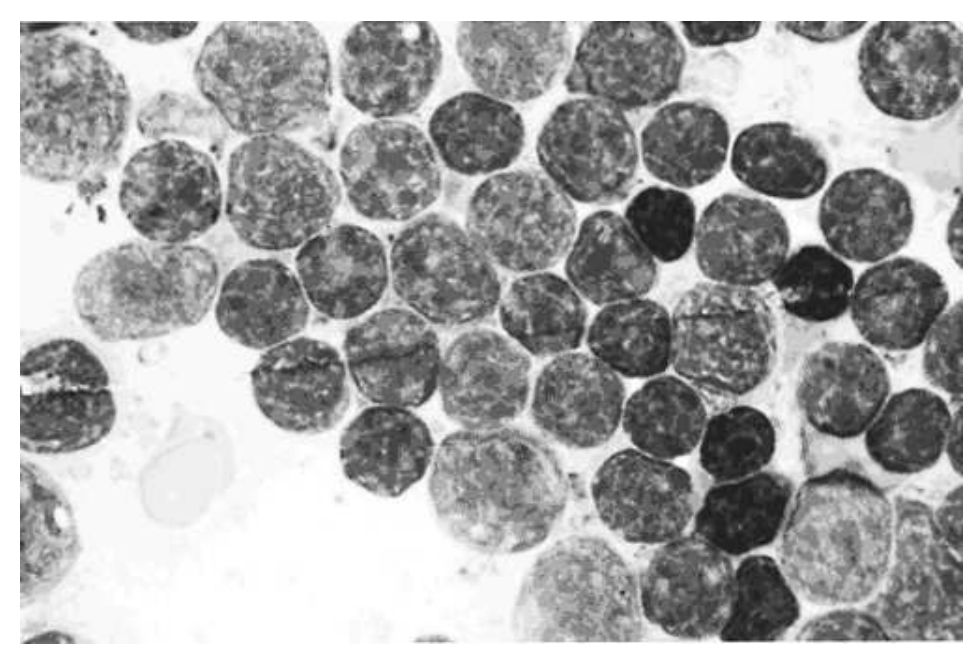

Fig. 1. Monochrome photomicrographic image of the footprint of lymphatic gland. Objective $\times 100$.

On the original RGB-images 4327 nuclei of lymphocytic cells important for diagnostics were indicated by experts. On the monochrome images the regions corresponding to the indicated nuclei were selected and further analyzed. 


\section{Selection and Extraction of Features for Lymphocyte Nuclei Description}

The principal property of the proposed technology is the generation of lymphocyte nuclei description by the features chosen and calculated from the images of specimens by the methods of image processing and analysis, and also by the methods of mathematical morphology and Fourier analysis. On the basis of obtained features, the cell nuclei were described by the vectors of numerical characteristics, which could be classified using pattern recognition techniques.

During the morphological analysis of lymphocyte nuclei, hematologists use the following features.

1. Nuclei size and density of different lymphoid cells in the specimen (presence of cells with nucleus larger than in most cells).

2. Nuclear form (round, oval, folded), presence of invaginations.

3. Textural features of chromatin (dispersed or condensed pattern; if dispersed - visual diameter of chromatin fibrils).

4. Presence or absence of nucleoli; if present - their number, size, form and location in nuclei (central or peripheral).

We provided formal equivalents of some of the above features. They can be translated into geometrical and textural features of cell nuclei. Thus, the following 47 features were chosen to describe nuclei morphology:

1) an area of nucleus in pixels;

2) four statistical features calculated on nucleus brightness histogram (average, dispersion, $3^{\text {rd }}$ and $4^{\text {th }}$ central moments);

3) 16 granulometric features of nucleus;

4) 26 features calculated on the Fourier spectrum of nucleus.

Formal approach for the description of the type of chromatin is the approximation of the size and the number of light oval-shaped or round regions of the nucleus. The following method is proposed to evaluate this information. Let the range of variation of a positive integer $k$ be determined, for example, $k=1,2, \ldots, n$. Consider a set of values $a_{1}, a_{2}, \ldots, a_{n}$ where $a_{k}$ is the difference between brightness masses of the original image and its opening with round structuring element of the radius $k$. Then, a size distribution $b_{1}, b_{2}, \ldots, b_{n}$ can be calculated, where $b_{1}=a_{1}, b_{k}=a_{k}-a_{k-1}, k=2, \ldots, n$. Since light regions of the source image are described by pixels with approximately equal values, $b_{k}$ can be interpreted as a measure of the relative number of light regions of the image that look like circles of the radius $k$.

Table 1 presents characteristics which were used to define the $3^{\text {rd }}$ and the $4^{\text {th }}$ groups of features of lymphocytic nuclei. The $3^{\text {rd }}$ group of features consists of characteristics 1-16 which were calculated on the set $b_{1}, b_{2}, \ldots, b_{n}$ (this set was considered as a range of some function of discrete argument $k$ ).

The $4^{\text {th }}$ group of features was obtained in the following way. As it is known, the Fourier spectrum is often used for texture analysis and description. The nucleus extracted in the monochrome image was placed against the black background (i.e., the background with zero gray level) and treated as a square image with dimension equal to some power of two. The Fast Fourier transform was used, then, to obtain a spec- 
trum of this image which was represented by the $2 N \times 2 N$ matrix $A$. Functions $F(r)$ and $F(\alpha)$ were considered for each nucleus. $F(r)$ is a sum of the matrix elements located on the semi-circle with the radius $r$ centered in the middle of matrix $A, r=0,1, \ldots, N$. $F(\alpha)$ is a sum of the matrix elements located on the line segment of the length $N$ originating from the middle of a matrix and forming the angle $\alpha$ with horizontal axis (anticlockwise), $\alpha \in[0, \pi]$. Table 1 presents the characteristics of functions $F(r)$ and $F(\alpha)$ (marked with the sign ' + ') which form the $4^{\text {th }}$ group of features. Since rotation of the nucleus causes a circular shift of corresponding $F(\alpha)$ plot, only six rotation-invariant characteristics from 20 presented in Table 1 were chosen to describe function $F(\alpha)$.

Table 1. Characteristics used to define the $3^{\text {rd }}$ and the $4^{\text {th }}$ group of features of lymphocytic nuclei. Granulometrical features were obtained by use of characteristics 1-16. Fourier features were obtained by use of characteristics of the functions $F(r)$ and $F(\alpha)$ marked with sign ' + '.

\begin{tabular}{|c|c|c|c|c|c|}
\hline Characteristic & $F(r)$ & $|F(\alpha)|$ & Characteristic & $F(r)$ & $F(\alpha)$ \\
\hline 1. Average & + & & $\begin{array}{l}\text { 11. Value of left local } \\
\text { maximum }\end{array}$ & + & \\
\hline 2. Dispersion & + & & $\begin{array}{l}\text { 12. Value of right local } \\
\text { maximum }\end{array}$ & + & \\
\hline 3. $3^{\text {rd }}$ central moment & + & & $\begin{array}{l}\text { 13. Number of local } \\
\text { minima }\end{array}$ & + & + \\
\hline 4. $4^{\text {th }}$ central moment & + & & $\begin{array}{l}\text { 14. Abscissa of global } \\
\text { minimum }\end{array}$ & + & \\
\hline 5. Number of local maxima & + & + & $\begin{array}{l}\text { 15. Abscissa of left local } \\
\text { minimum }\end{array}$ & + & \\
\hline $\begin{array}{l}\text { 6. Abscissa of global maxi- } \\
\text { mum }\end{array}$ & + & & $\begin{array}{l}\text { 16. Abscissa of right } \\
\text { local minimum }\end{array}$ & + & \\
\hline $\begin{array}{l}\text { 7. Abscissa of left local } \\
\text { maximum }\end{array}$ & + & & 17. Sum of local minima & + & + \\
\hline $\begin{array}{l}\text { 8. Abscissa of right local } \\
\text { maximum }\end{array}$ & + & & $\begin{array}{l}\text { 18. Value of global } \\
\text { minimum }\end{array}$ & + & + \\
\hline 9. Sum of local maxima & + & + & $\begin{array}{l}\text { 19. Value of left local } \\
\text { minimum }\end{array}$ & + & \\
\hline $\begin{array}{l}\text { 10. Value of global maxi- } \\
\text { mum }\end{array}$ & + & + & $\begin{array}{l}\text { 20. Value of right local } \\
\text { minimum }\end{array}$ & + & \\
\hline
\end{tabular}

\section{Cluster Analysis: Experimental Check of the Suggested Information Technology}

To preprocess the images of the specimens and to calculate the nuclei features, a program was developed which uses the libraries of the "Black Square" software system $[3,4]$. After calculation of the features, their statistical and qualitative analysis was carried out: 
- feature variances and pair correlations were calculated;

- groups of features were defined such that, in each group, pair correlation of the features was no greater than 0,$9 ; 0,8 ; \ldots ; 0,1$.

As a result, 9 groups of features with small variance were defined, as well as 9 groups of features with large variance. The "robustness" of the nuclei features was investigated, i.e., we evaluated to what extent the changes in the nuclei contour caused changes in values of its features. Conducted analysis allowed us to select several groups of features, which further yielded diagnostically-perspective taxonomies.

The main results of the lymphocytic cells investigations are based on their cluster analysis using developed feature space and its subspaces. There are many ways to identify clusters in data sets, in our work we used FOREL algorithm [2]. It has one parameter, the radius $R$ of a separating ball, and operates as follows. The set of objects we have to analyze may be considered as a set of points $P_{1}, P_{2}, \ldots, P_{n}$ in a multidimensional feature space with some metric. In this space a separating ball is considering with radius $R$ centered in arbitrary point $P_{i}$. A set $M$ is defining as a set of all points located in the ball. An auxiliary point $C$ is considering with coordinates equal to the mean coordinates of the points from the set $M$. If point $C$ does not coincide with the point $P_{i}$, the center of the ball is shifted to $C$. Then, the set $M$ is defined again and a new position for the center of the ball is computed. Thus the ball is moved to the local concentration of points until on some iteration its new position will coincide with the previous one. When it happens, points of the set $M$ are all put in the new cluster and excluded from the further consideration. This process is repeated until all points $P_{1}, P_{2}, \ldots, P_{n}$ will be put in clusters.

A number of sets of clusters were obtained by using different sets of features and different radii of the separating ball, including:

- all 47 features;

- 24 features with the numbers $1-14,16-19,21,26-28,32,36$ (features with large variance and pair correlations no greater then 0,9 );

- 18 features with the numbers 1-3, 6-8, 10-14, 16, 19, 26-28, 32, 36 (features with large variance and pair correlations no greater then 0,9 , excluding "non-robust" features).

Obtained sets of clusters were evaluated using various criteria (e.g., the number and the size of clusters in each set, the character of nuclei distribution in large clusters, etc.). Several "interesting" sets of clusters were selected (they were obtained by using groups of 24 and 18 features described above) which have good formal characteristics of their clusters and are promising for interpretation by hematologists. For example, 58 clusters were identified when we used 18 features described above and $R=0,48$. Among these clusters, 23 contain only one nucleus and five large clusters contain 257-1727 nuclei each and more than $88 \%$ of all nuclei together. The interesting fact about these five clusters is that if we order them by the mean square of cluster's nuclei, then the fraction of the nuclei of the patients with aggressive lymphoid tumors in the cluster is increasing when mean area of the cluster's nuclei is increasing.

Experiments were conducted to form and investigate feature sets and to develop decision rules for classifying the patients as being the members of one of the two classes, corresponding to two types of lymphoid tumors (aggressive lymphoid tumors and indolent chronic lymphocytic leukemia). 
These experiments allowed us to suggest a new method to form feature description of the patient. By using the results of cluster-analysis of cell nuclei, each patient is described by the features calculated as relational numbers of patient's nuclei belonging to the large clusters of the clusterization. Preliminary experiments show that good classification results can be obtained in this feature space.

The obtained results show that:

1) the set of diagnostically important nuclei of the patients with considered lymphoid tumors is substantially heterogeneous since different clusters in it were clearly identified;

2) clusterization of the lymphocytic nuclei using developed feature set is important from the medical standpoint;

3) formal nucleus characterization by the developed feature set corresponds well with its qualitative morphological description and serves as a basis for development the automated software systems of morphological analysis of the blood cells and diagnosis of hemoblastoses;

4) the suggested technology provides transition from the diagnostic analysis of lymphocytes nuclei to diagnosing patients with hematopoietic tumors by the use of pattern recognition techniques.

\section{Conclusion}

The information technology for the automated morphological analysis of the images of lymphoid cell nuclei of patients with hematopoietic tumors based on the combined use of pattern recognition and image analysis techniques is described. This technology provides a transition from the diagnostic analysis of lymphocytes nuclei to the diagnosing patients with hematopoietic tumors by means of pattern recognition techniques. Experimental check of the technology shows that it can be successfully used in software systems for automated diagnosis of the hematopoietic tumors.

In the future, we plan to augment the image database by the new images of specimens of lymphatic tissues. We also plan to develop another version of information technology for the morphological analysis of the lymphoid cell nuclei of patients with hematopoietic tumors by using efficient pattern recognition algorithms based on estimate calculation which could be directly applied to the images and its fragments [5]. This technology would allow to exclude feature models of images from recognition process.

\section{References}

1. Churakova, J.V., Gurevich, I.B., Hilkov, A.V., Jernova, I.A., Kharazishvili, D.V., Nefyodov, A.V., Sheval, E.V.: Selection of Diagnostically Valuable Features for Morphological Analysis of Blood Cells. Pattern Recognition and Image Analysis: Advances in Mathematical Theory and Applications. 2 (2003) $382-383$ 
2. Elkina, V.N., Zagoruiko, N.G.: Some Classification Algorithms Developed at Novosibirsk. In: Simon, J.C. (ed.): Intelligence Artificielle, Reconnaissance des Formes. R.A.I.R.O. Informatique/Computer Science. 1 (1978) 37-46

3. Gurevich, I.B., Khilkov, A.V., Murashov, D.M., et al.: Black Square Version 1.0: Programm Development System for Automation of Scientific Research and Education. Pattern Recognition and Image Analysis: Advances in Mathematical Theory and Applications. 4 (1999) 609-634

4. Gurevich, I.B., Murashov, D.M., Nefyodov, A.V., Hilkov, A.V.: Extraction of Diagnostic Features of Hematopoietic Tumors from the Images of Specimens by Software System "Black Square". Proceedings of the 6th Russian Conference "Methods and Means of the Complex Graphic Data Processing", Nizhniy Novgorod, Russian Federation, September 25-27, (2001) 74-75 (in Russian)

5. Gurevich, I.B., Nefyodov, A.V.: Algorithms for Estimate Calculations Designed for 2D Support Sets. Part 1: Rectangular Support Sets. Pattern Recognition and Image Analysis: Advances in Mathematical Theory and Applications. 4 (2001) 662-689

6. Zhuravlev, Yu.I., Gurevitch, I.B.: Pattern Recognition and Image Recognition. Pattern Recognition and Image Analysis: Advances in Mathematical Theory and Applications in the USSR. 2 (1991) 149-181 